\section{Mit halber Substanz, aber voller Kraft in die Pollensaison}

s eit Anfang des Jahres befindet sich ein neuer $\mathrm{H}_{1}$-Rezeptor-Antagonist auf dem Markt, der zur Behandlung allergischer Rhinitiden sowie der chronischen Urtikaria zugelassen ist: Levocetirizin $\left(\mathrm{Xusal}^{\circledR}\right)$, die aktive Komponente von Cetirizin. Dieses bewährte Antihistaminikum der zweiten Generation liegt nämlich als Razemat, also als Gemisch zweier spiegelbildlicher Isomere (Enantiomere) vor, von denen sich das Dextrocetirizin als inaktiv erwiesen hat - für die Antihistamin-Wirkung ist ausschließlich Levocetirizin verantwortlich.

In präklinischen Versuchen stellte sich heraus, dass Levocetirizin in vitro eine zweimal höhere Affinität zum $\mathrm{H}_{1}$ Rezeptor besitzt als Cetirizin, zudem bleibt es erheblich länger daran gebunden. Da die Verbindung praktisch nicht verstoffwechselt wird, sind Interaktionen mit anderen Medikamenten un- wahrscheinlich. Im EKG fand man bisher keine relevanten Veränderungen des QT-Intervalls.

Wie Alexander Kapp (Hannover) ausführte, erwies sich Levocetirizin in einer vergleichenden Untersuchung mehrerer moderner $\mathrm{H}_{1}$-Blocker als potentester Vertreter seiner Klasse: In der doppelblinden, randomisierten Crossover-Studie prüfte man Wirkstärke, Zuverlässigkeit und Wirkdauer nach Histamin-Provokation an gesunden Probanden. Dabei hemmte Levocetirizin Quaddelbildung und Hautrötung ausgeprägter als die Vergleichspräparate: Bei der Mehrzahl der Probanden reduzierte es die Hautreaktion über einen Zeitraum von 2 bis 12 Stunden um über 95\%. Kapp lobte insbesondere das relativ konstante Ansprechen praktisch aller Behandelten bei nur geringer interindividueller $\mathrm{Va}$ riabilität.

\section{Immuntherapie - je früher desto besser}

D ie spezifische Immuntherapie reduziert bei allergischem Asthma bronchiale die allergenspezifische und auch unspezifische Hyperreagibilität, die Symptomatik und den Verbrauch an Bedarfsmedikamenten. Das zumindest bestätigte eine Metaanalyse von $62 \mathrm{Stu}-$ dien, in der die Therapieerfolge der spezifischen Immuntherapie bei Asthma belegt wurden.

Allerdings sollte eine Immuntherapie in einem möglichst frühen Asthmastadium begonnen werden. Denn zu Beginn des allergischen Asthma bronchiale spielen Inhalationsallergene noch eine wichtige Rolle in der Krankheitspathogenese, im späteren Verlauf tritt die Allergie mehr in den Hintergrund. Die Hyperreagibilität wird zunehmend unspezifisch, die Entzündung der Atemwege chronifiziert und das Gewebe wird irreversibel geschädigt. Die wichtigste Zielgruppe für eine spezifische Immuntherapie sind daher Asthmatiker mit

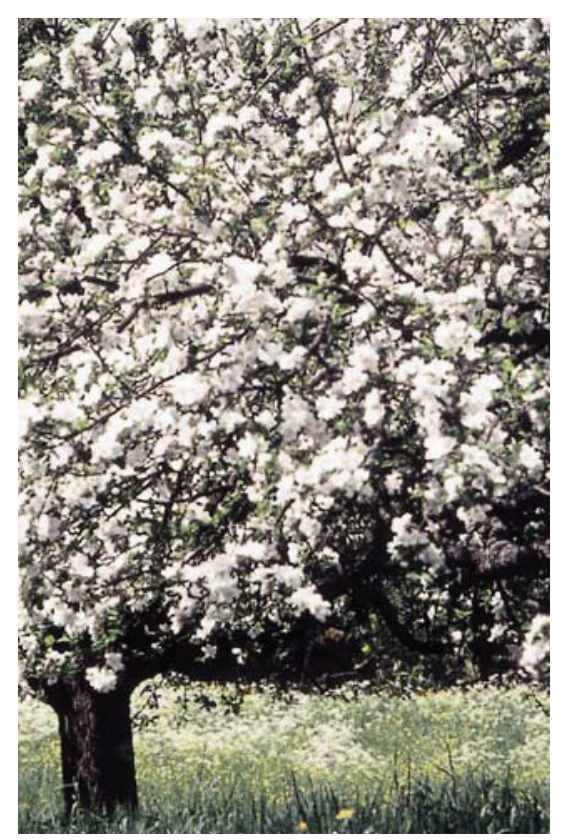

Früh eingesetzt kann die spezifische Immuntherapie Allergikern zu unbeschwerten Frühlingsgefühlen verhelfen.
Eine weitere Studie belegte den guten Antipruritus-Effekt des Arzneimittels: Der provozierte Juckreiz der Testpersonen wurde um $91 \%$ gemildert. Kapps knappes Fazit: „Levocetirizin ist in halber Dosierung mindestens genauso wirksam wie Cetirizin."

Als das aktive Element von Cetirizin sollte Levocetirizin auch antientzündlich wirken; in der Tat ließ sich das im Hautkammermodell, also in vivo, belegen: Die Migration eosinophiler Granulozyten war ebenso wie die Expression von VCAM-1 in Anwesenheit der Verbindung signifikant vermindert.

Deutliche Linderung verspricht die Substanz ferner bei der saisonalen wie der ganzjährigen allergischen Rhinitis. Insbesondere auch die besonders belastende Histamin-induzierte Erhöhung des nasalen Widerstands scheint sie wirksam zu bekämpfen.

wpa

Einführungspressekonferenz „Xusa $\left.\right|^{\circledR}$ die neue Cetirizin-Generation“, München, 2001. Veranstalter: UCB-Pharma.

mildem bis mittelschwerem Asthma, die gleichzeitig unter einer Rhinitis leiden und bei denen die Krankheitsprozesse noch nicht zu weit fortgeschritten sind.

Der Erfolg einer spezifischen Immuntherapie hängt entscheidend von der Auswahl der Patienten, den eingesetzten Allergenextrakten und ihrer exakten Dosierung ab. Die Therapie ist effektiv bei der Behandlung von Rhinitis und Asthma bei Pollen-, Hausstaubmilben- und Katzenhaarallergie, wenn der richtige Extrakt gegeben wird, stellte J. Bousquet aus Montpellier, Frankreich, im Oktober auf dem PneumologenSymposium "Allergie und Asthma“ in Dresden fest. Als optimale Dosis für die Erhaltungstherapie gelten Majorallergen-Mengen von 5-10 mg, wobei die verträgliche Dosis jeweils individuell ermittelt werden muss.

Nach Informationen von ALK-Scherax

Arzneimittel. 